Supporting Information

\title{
Boronate Affinity Fluorescent Nanoparticles for Förster Resonance Energy Transfer Inhibition Assay of cis-Diol Biomolecules
}

Shuangshou Wang, Jin Ye, Xinglin Li, and Zhen Liu*

State Key Laboratory of Analytical Chemistry for Life Science, School of Chemistry and

Chemical Engineering, Nanjing University, Nanjing 210023, China

* Corresponding author: Dr. Zhen Liu. Tel/Fax: +86 2589685639

Email: zhenliu@nju.edu.cn

\section{Table of contents}

- Supplementary Figures

Figure S1 to Figure S8

- Supplementary Tables

Table S1 


\section{Supplementary Figures}

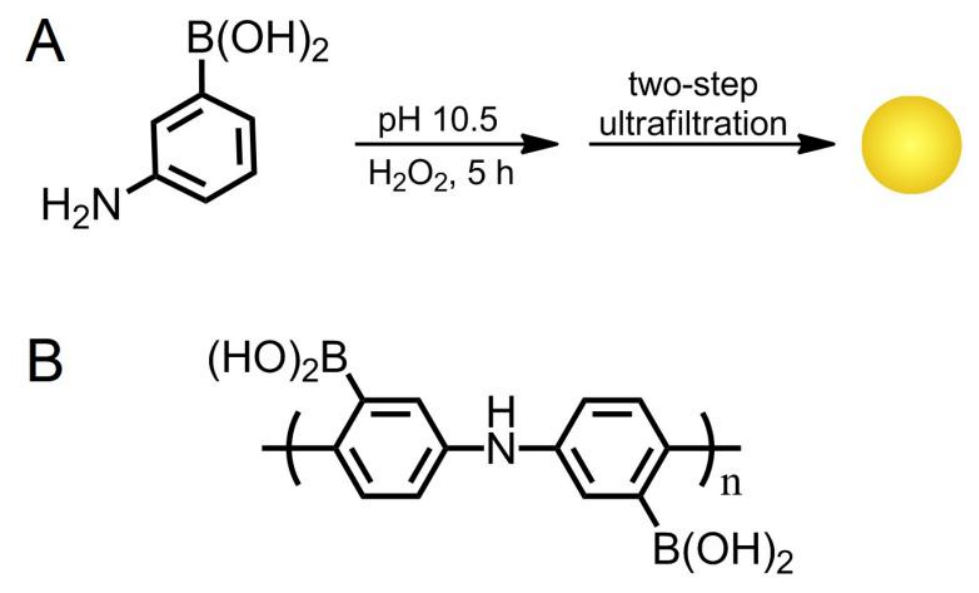

Scheme S1. The synthesis route (A) and possible chemical structure (B) of poly(mAPBA) NPs. 

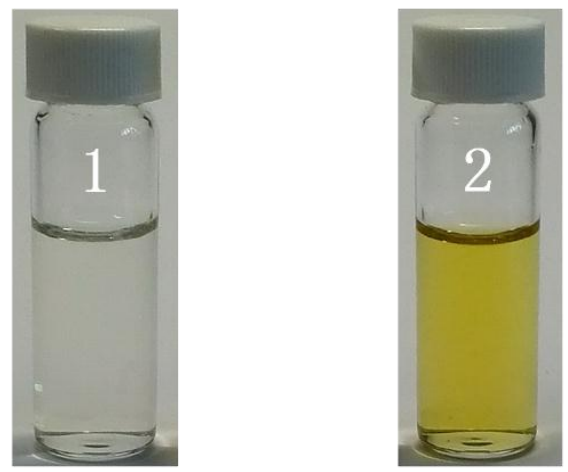

Figure S1. Pictures of aqueous solution mAPBA (1) and poly(mAPBA) NPs (2) (concentration: $2 \mathrm{mg} / \mathrm{mL}$ ). 

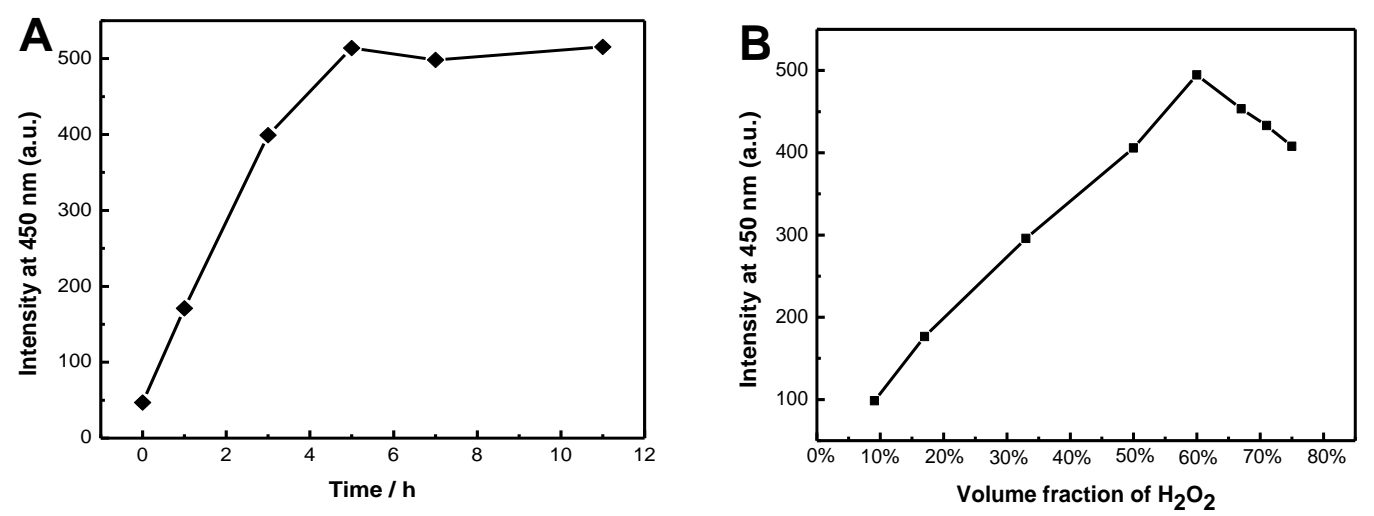

Figure S2. A) Fluorescence intensity of poly(mAPBA) NPs prepared with different polymerization times; B) Fluorescence intensity of poly(mAPBA) NPs prepared at different volume fractions of hydrogen peroxide (polymerization time was set at $5 \mathrm{~h}$ ). 

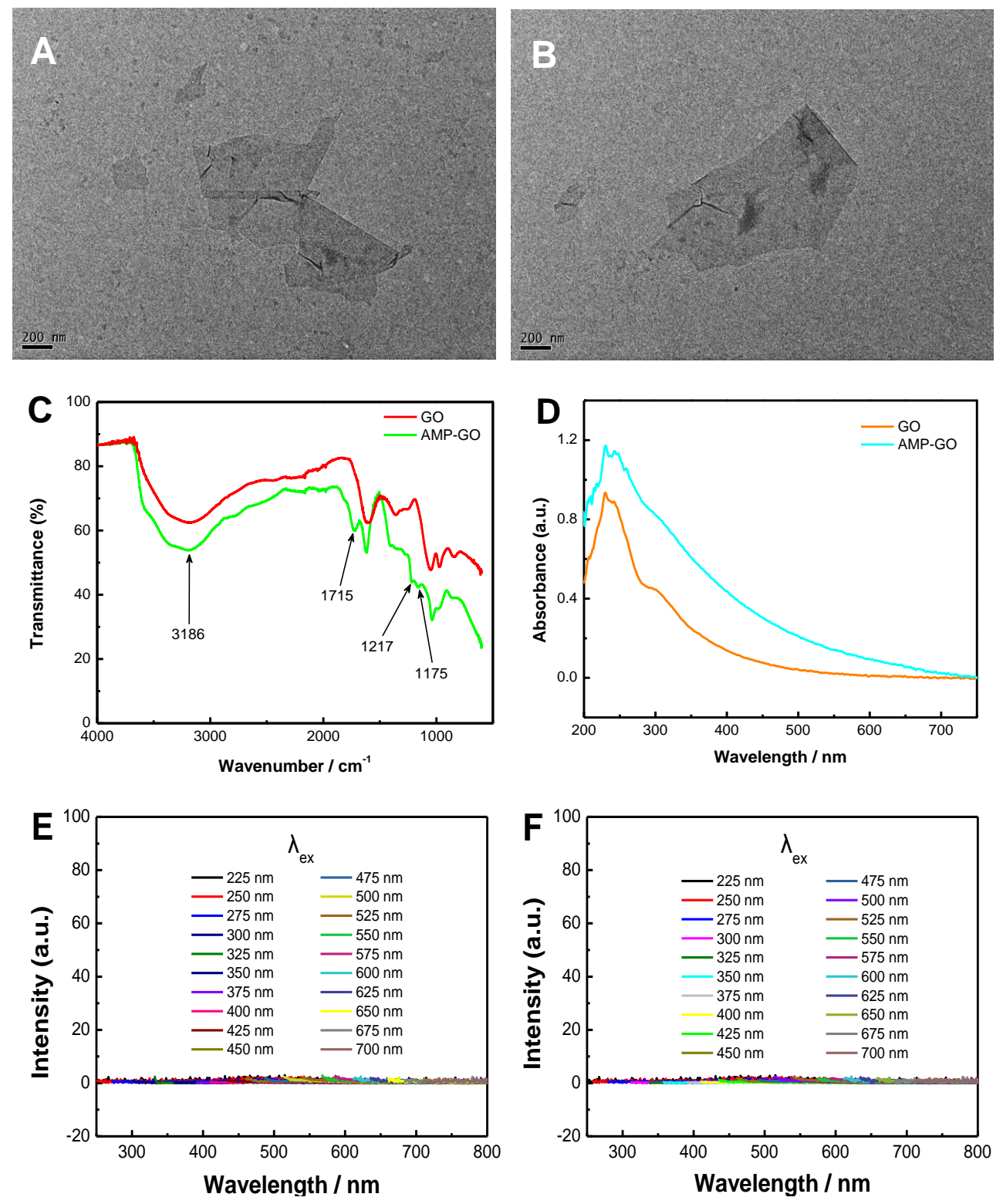

Figure S3. A) TEM image of GO; B) TEM image of AMP-GO; C) FT-IR spectrum of GO and AMP-GO (3186 cm $\mathrm{cm}^{-1}$ :-OH; $\left.1715 \mathrm{~cm}^{-1}: \mathrm{C}=\mathrm{N} ; 1217 \mathrm{~cm}^{-1}, 1175 \mathrm{~cm}^{-1}: \mathrm{P}=\mathrm{O}\right)$; D) UV-vis absorption spectrum of GO and AMP-GO; E) Fluorescence spectrum of GO under different excitation wavelength; F) Fluorescence spectrum of AMP-GO under different excitation wavelength. 

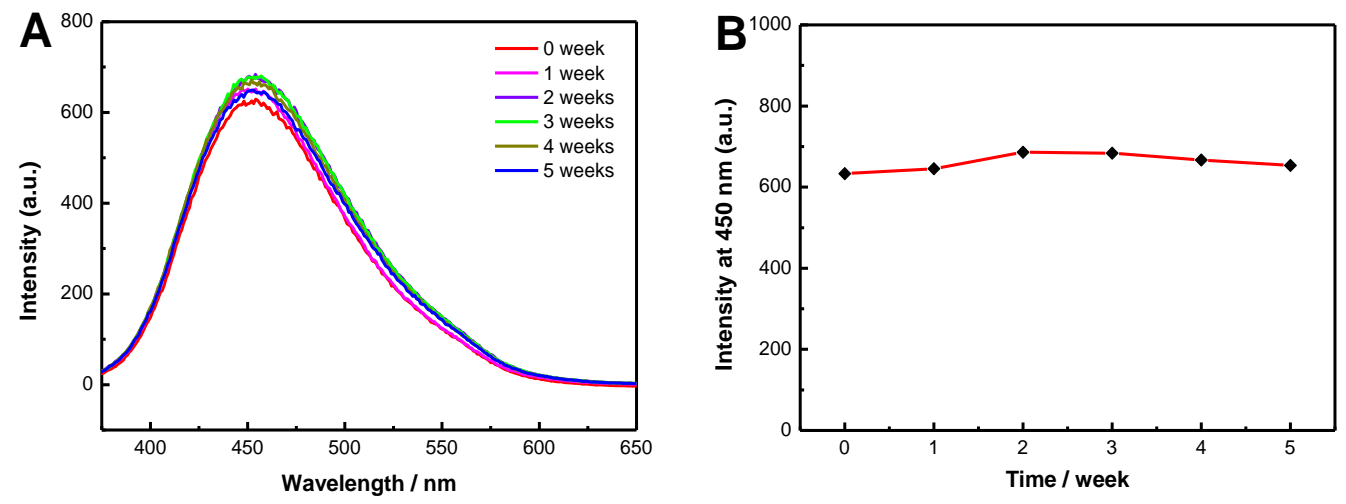

Figure S4. A) Fluorescence spectra of poly(mAPBA) NPs at different storage times at room temperature; B) Fluorescence intensity of poly(mAPBA) NPs as a function of storage time. 

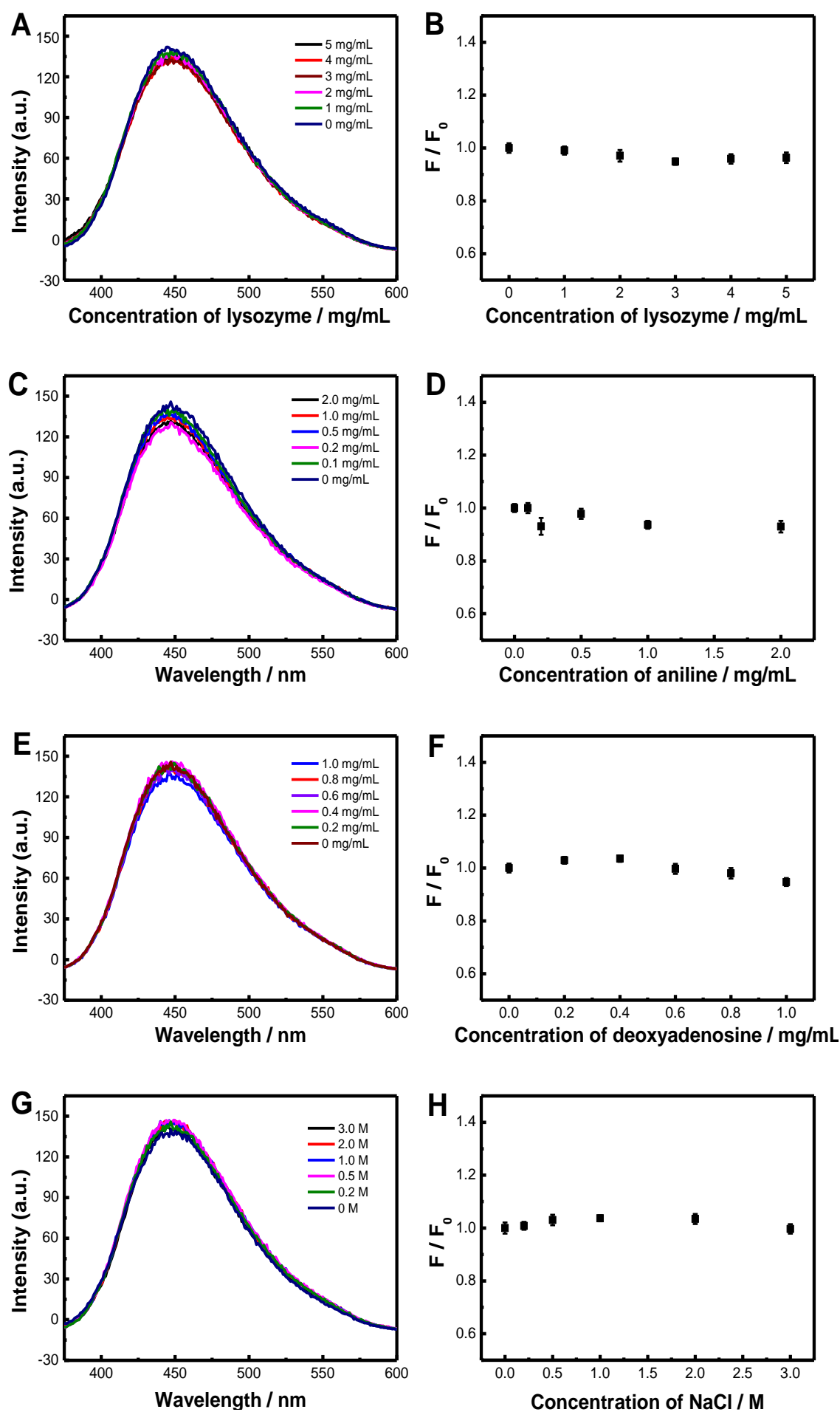

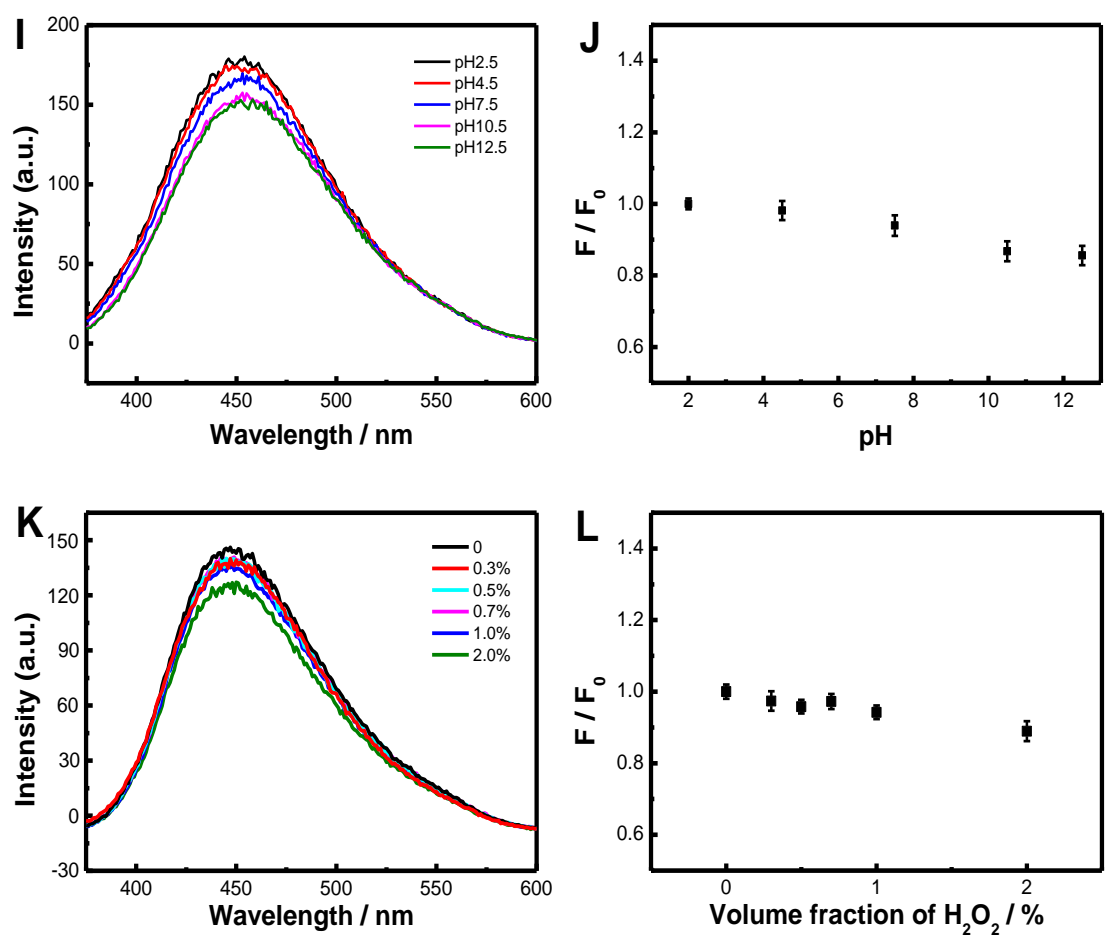

Figure S5. Fluorescence intensity of poly(mAPBA) NPs/AMP-GO conjugates in the presence of different environmental factors. A, B: lysozyme; C, D: aniline; E, F: deoxyadenosine; G, $\mathrm{H}: \mathrm{NaCl}$; I, J: pH; K, L: $\mathrm{H}_{2} \mathrm{O}_{2}$. 

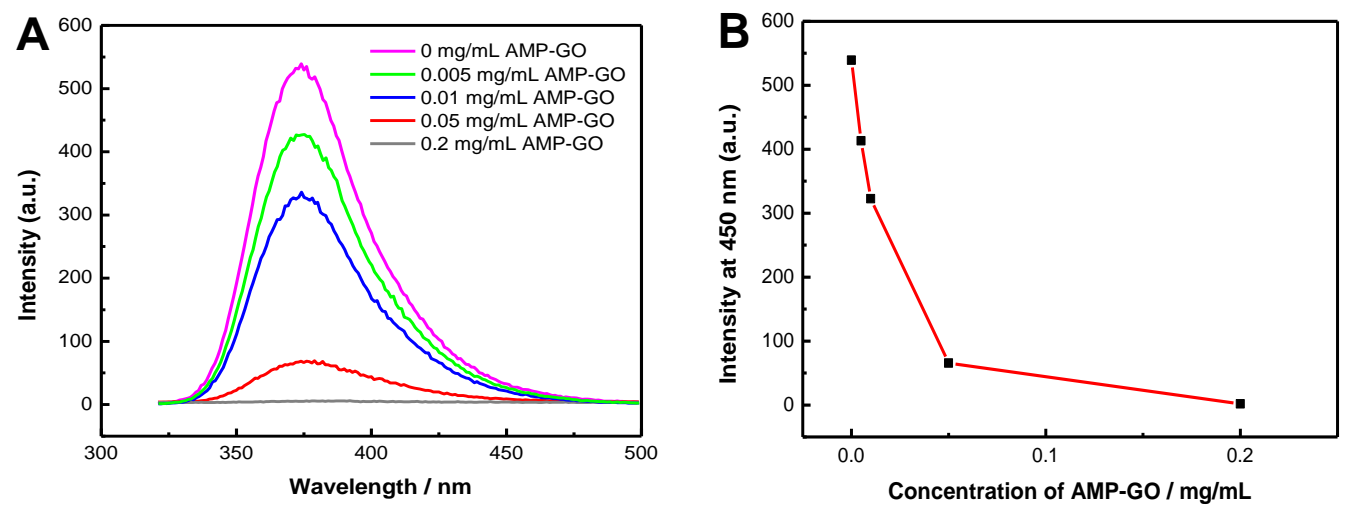

Figure S6. A) Fluorescence emission spectra of mAPBA monomer with the presence of AMP-GO of different concentration; B) Dependence of the fluorescence intensity of mAPBA monomer on the concentration of AMP-GO. 


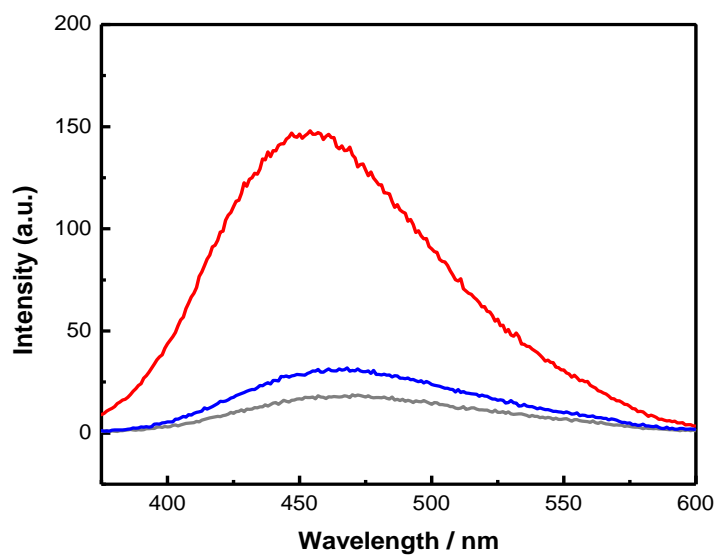

Figure S7. Fluorescence quenching of poly(mAPBA) NPs by AMP-GO and GO. Red: poly(mAPBA) NPs; blue: poly(mAPBA) NPs/GO conjugates; gray: poly(mAPBA) NPs/AMP-GO conjugates. Concentration: $0.25 \mathrm{mg} / \mathrm{mL}$ for poly(mAPBA) NPs; $0.2 \mathrm{mg} / \mathrm{mL}$ for AMP-GO and GO, respectively. The excitation wavelength was set at $350 \mathrm{~nm}$. 


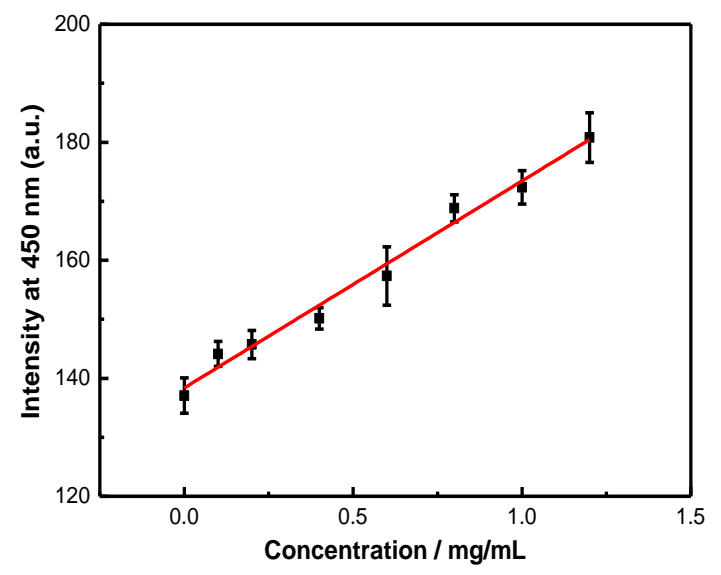

Figure S8. Fluorescence intensity of poly(mAPBA) NPs/AMP-GO conjugates as a function of concentration of glucose at $\mathrm{pH} 7.0\left(R^{2}=0.977, \mathrm{n}=8\right)$. 


\section{Supplementary Tables}

Table S1. Energy dispersive X-Ray spectroscopy (EDX) analysis for elements of GO and AMP-GO.

\begin{tabular}{ccccc}
\hline \multirow{2}{*}{ Element } & \multicolumn{3}{c}{ GO } & \multicolumn{2}{c}{ AMP-GO } \\
\cline { 2 - 5 } & $\mathrm{Wt} \%$ & $\mathrm{At} \%$ & $\mathrm{Wt} \%$ & $\mathrm{At} \%$ \\
\hline $\mathrm{C}$ & 74.46 & 79.52 & 80.73 & 84.21 \\
$\mathrm{~N}$ & 00.00 & 00.00 & 03.98 & 03.57 \\
$\mathrm{O}$ & 25.54 & 20.48 & 15.29 & 12.22 \\
\hline
\end{tabular}

